\title{
A Surfactant Jacket for Carbon Nanotubes
}
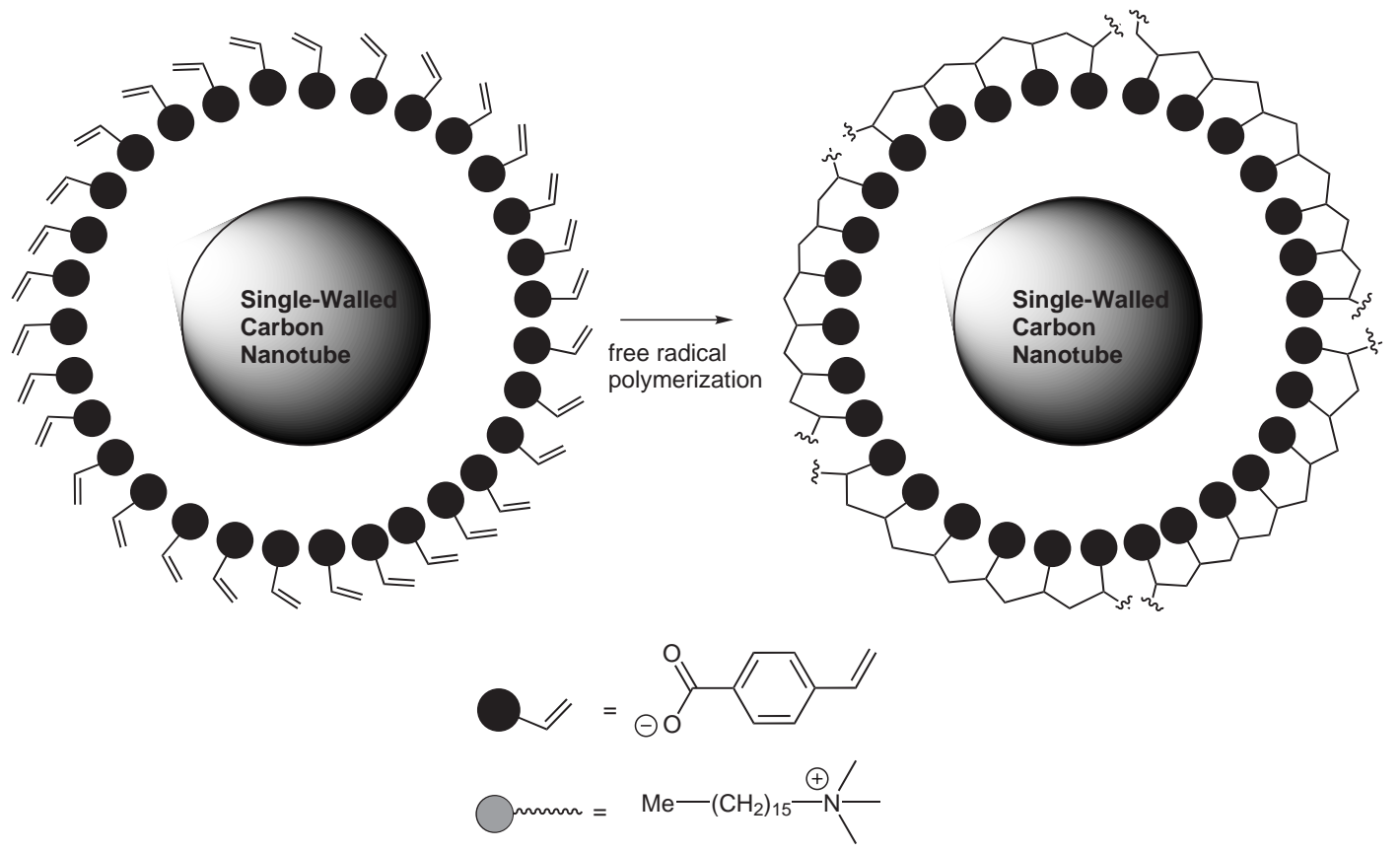

Significance: Carbon nanotubes are emerging as one of the most versatile new material classes for the design of sensors, electronics, and highstrength materials. Strong inter-nanotube attractions lead to bundled structures that are insoluble and considerable efforts have been made to create robust dispersions of these materials. Dispersions in water are attractive for biological applications, however, systems with simple surfactants have limited stability and shelf life. In this contribution a polymerizable ionic complex was bound to the carbon nanotubes and subsequent polymerization created superior dispersions.
Comment: The polymerization of an electrostatic surfactant complex opens a number of modular schemes for creating functionalized carbon nanotube dispersions. Functionalized comonomers that enable bioconjugation or installation of ligands on the surface. The authors performed rigorous scattering studies to prove that the dispersions contained completely unbundled dispersions, that lacked crosslinking between individual tubes. Such well-defined and -behaved systems are sure to find applications. 